\title{
Directed flow and freeze-out in relativistic heavy-ion collisions at NICA and FAIR energies
}

\author{
L.V. Bravina* \\ Department of Physics, University of Oslo, Oslo, Norway \\ Skobeltzyn Institute of Nuclear Physics, Moscow State University, Moscow, Russia \\ E-mail: larissa.bravina@fys.uio.no
}

Yu. Kvasiuk

Taras Shevchenko National University of Kyiv, Kyiv, Ukraine

\section{Sachenko}

Taras Shevchenko National University of Kyiv, Kyiv, Ukraine

\section{S. Yu. Sivoklokov}

Skobeltzyn Institute of Nuclear Physics, Moscow State University, Moscow, Russia

\section{O. Vitiuk}

Taras Shevchenko National University of Kyiv, Kyiv, Ukraine

\section{E.E. Zabrodin}

Department of Physics, University of Oslo, Oslo, Norway

Skobeltzyn Institute of Nuclear Physics, Moscow State University, Moscow, Russia

\begin{abstract}
Directed flow of charged particles is studied in nucleus-nucleus collisions simulated within the energy range accessible for NICA and FAIR facilities. Two transport cascade models, UrQMD and QGSM, are employed. These models use different mechanisms of the string excitation and string fragmentation. Despite of the differences, directed flows of charged pions and charged kaons in both models remain antiflow-oriented with reduction of the collision energy from $\sqrt{s}=$ 11.5 GeV to $3.5 \mathrm{GeV}$. In contrast, the directed flow of protons changes its sign from antiflow to normal flow within the investigated energy interval. Both models favor continuous non-uniform emittence of hadrons from the expanding fireball rather than sharp, or sudden, freeze-out picture adopted by majority of hydrodynamic models. We found that the earlier frozen hadrons carry the strongest directed flow at midrapidity, although the flow development even at $|y| \leq 0.5$ takes about 8-12 fm/c for different hadron species.
\end{abstract}

Corfu Summer Institute 2018 "School and Workshops on Elementary Particle Physics and Gravity" (CORFU2018)

31 August - 28 September, 2018

Corfu, Greece

${ }^{*}$ Speaker. 


\section{Introduction}

Search for collectivity in both small and big hot and dense systems, created in e.g. protonproton or heavy-ion collisions at relativistic energies, is a modern trend in high energy physics nowadays. One of the most prominent signals to study is the (collective) flow of hadrons in the transverse plane of the collision. Decomposition of the transverse flow into Fourier series was proposed in $[1,2]$. It casts the invariant cross section as

$$
E \frac{d^{3} N}{d^{3} p}=\frac{d^{2} N}{\pi d p_{T}^{2} d y}\left\{1+2 \sum_{n=1}^{\infty} v_{n} \cos \left[n\left(\phi-\Psi_{n}\right)\right]\right\}
$$

where

$$
v_{n}=\left\langle\cos \left[n\left(\phi-\Psi_{n}\right)\right]\right\rangle .
$$

In Eq.(1.1) $p_{T}$ and $y$ denotes particle transverse momentum and rapidity, respectively, $\phi$ is the azimuth between the $\overrightarrow{p_{T}}$ and the participant event plane, and $\Psi_{n}$ is the azimuthal angle of the event plane of $n$-th flow component. The unity in curly brackets stands for the isotropic flow, and the infinite sum represents the anisotropic flow. Its components, presented by Eq.1.2), are dubbed as directed, $v_{1}$, elliptic, $v_{2}$, triangular, $v_{3}$, flow, and so forth. Note, that averaging in this formula is performed over all particles in every event and over all events in the data sample. It is worth noting that up to middle of 1990s the standard analysis of the collective flow of hadrons [3] has assumed the flow decomposition into the bounce-off flow (sometimes also called directed flow), elongated in the reaction plane, and the squeeze-out flow, developed along the normal to the reaction plane. According to definition, the bounce-off flow is the averaged projection of particle momentum on the impact parameter axis, $\left\langle p_{x} / A\right\rangle$, whereas the directed flow is represented by the averaged patio of $p_{x}$ to the hadron transverse momentum, $\left\langle p_{x} / p_{T}\right\rangle$. Therefore, the dependencies of both, bounceoff and directed, flows on rapidity, transverse momentum or centrality of the collision should be qualitatively similar.

One of the reasons to study directed flow in heavy-ion collisions is the sensitivity of this signal to the creation of even small amounts of quark-gluon plasma (QGP). Here the disappearance of the pressure gradients in the mixed phase with QGP and hadrons should lead to the so-called softest point of the equation of state [4]. The softening leads to the significant reduction or even disappearance of the directed flow $[5,6,7]$. Note, that at energies $E_{l a b}$ below $1-2 \mathrm{GeV} / c$ it was found that the flow of protons had a linear slope at midrapidity. At ultrarelativistic energies, corresponding to that at RHIC and LHC, directed flow of protons at midrapidity range seems to be very close to zero, whereas $v_{1}$ of charged pions and charged kaons is elongated in the antiflow direction. - We usually call the direction of the directed flow normal if the product $p_{x} \cdot y$ is positive, $p_{x} \cdot y>0$, and antiflow in the opposite case, if $p_{x} \cdot y<0[8,6]$. The study of low- and intermediate energy range within the beam energy scan (BES) program at RHIC led to "rediscovery" of the flip of proton directed flow from weak antiflow to the normal flow $[9,10]$. Does it mean that we have found the evidence of the first order deconfinement phase transition? Not necessarily, unfortunately, because microscopic transport models predicted such behavior of the proton directed flow quite long ago $[8,11,13]$.

In the present paper we will focus on the properties of the directed flow in heavy-ion collisions provided by Monte Carlo transport models. Brief description of both models, employed for our 
study, is given in Sec. 2. Section 3 presents main results of the energy scan for the directed flow of different hadron species and elaborates on the features of the flow development and evolution in the transport models. We show that the evolution of $v_{1}$ is closely connected to the freeze-out of hadrons. In microscopic models the freeze-out is continuous and does not resemble the sharp particle freeze-out adopted e.g. in many hydrodynamic models. Conclusions are drawn in Sec. 4.

\section{Main features of microscopic models}

The Ultra-relativistic Quantum Molecular Dynamics model (UrQMD) [14, 15] and QuarkGluon String Model (QGSM) [16, 17] are Monte-Carlo event generators designed for the description of hadron-hadron, hadron-nucleus, and nucleus-nucleus collisions in a broad energy range from hundred $\mathrm{MeV}$ up to several $\mathrm{TeV}$. As independent degrees of freedom UrQMD contains 55 baryon and 32 meson states, together with their antiparticles and explicit isospin-projected states, with masses up to $2.25 \mathrm{GeV} / c$. QGSM includes octet and nonet vector and pseudoscalar mesons, and octet and decuplet baryons, and their antiparticles. At lower energies the interaction dynamics of $h h$ or $A+A$ collisions takes into account interactions between the hadrons and their excited states, resonances. At higher energies both models treat the production of new particles via formation and fragmentation of specific colored objects, strings. Strings are uniformly stretched between the quarks, diquarks and their antistates with constant string tension $\kappa \approx 1 \mathrm{GeV} / \mathrm{fm}$. The excited strings are fragmenting into pieces via the Schwinger mechanism of $q \bar{q}$-pair production, and the distribution of newly produced hadrons is uniform in the rapidity space.

UrQMD utilizes the longitudinal mechanism of string excitation, where the string masses arise from momentum transfer. In contrast, QGSM treats the elementary hadronic interactions on the basis of Gribov-Regge theory. The model is based on the $1 / N_{c}$ (where $N_{c}$ is the number of quark colors or flavors) topological expansion of the amplitude for processes in quantum chromodynamics and string phenomenology of particle production in inelastic binary collisions of hadrons. Here strings are stretching between the constituents belonging to different hadrons. Both models employ tables of the experimentally available information, such as hadron cross sections, resonance widths and decay modes. If this information is lacking, the one-boson exchange model, detailed balance considerations and isospin symmetry conditions are used. The propagation of particles is governed by Hamilton equation of motion, and hadronic cascade is used for the description of $h A$ and $A+A$ interactions. Due to the uncertainty principle newly produced particles can interact further only after a certain formation time. Hadrons containing the valence quarks, however, can interact immediately with the reduced cross section $\sigma=\sigma_{q N}$. The Pauli principle is taken into account via the blocking of the final state, if the outgoing phase space is occupied.

\section{Results}

To investigate the basic features of the directed flow of pions and protons in UrQMD and QGSM we choose four bombarding energies, $\sqrt{s}=3.5 \mathrm{GeV}, 5.5 \mathrm{GeV}, 7.7 \mathrm{GeV}$ and $19.6 \mathrm{GeV}$, two systems of colliding nuclei, $\mathrm{Au}+\mathrm{Au}$ and $\mathrm{Xe}+\mathrm{Xe}$ (as planed for NICA), and three centrality intervals, proposed by the STAR collaboration, $\sigma / \sigma_{g e o}=0-10 \%, 10-40 \%$ and $40-80 \%$. The material presented below has been partially shown at the conferences QUARKS'18 [18] and ICNFP'2018 


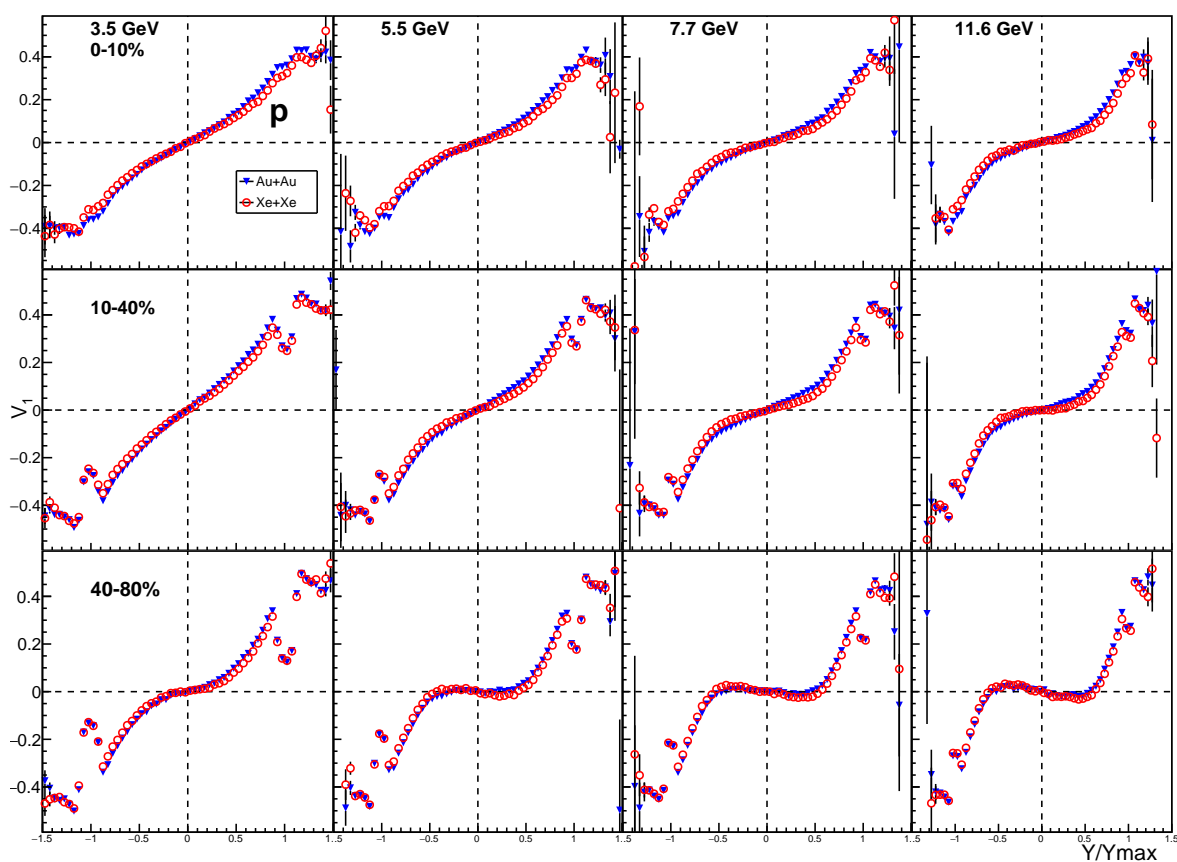

Figure 1: Directed flow of protons vs rapidity in QGSM calculations of $\mathrm{Au}+\mathrm{Au}$ (triangles) and $\mathrm{Xe}+\mathrm{Xe}$ (open circles) collisions at $\sqrt{s}=3.5 \mathrm{GeV}$ (first left column), 5.5 GeV (second column), 7.7 GeV (third column) and $19.6 \mathrm{GeV}$ (fourth column) with centrality $0-10 \%$ (upper row), $10 \%-40 \%$ (middle row) and $40 \%-80 \%$ (bottom row), respectively.

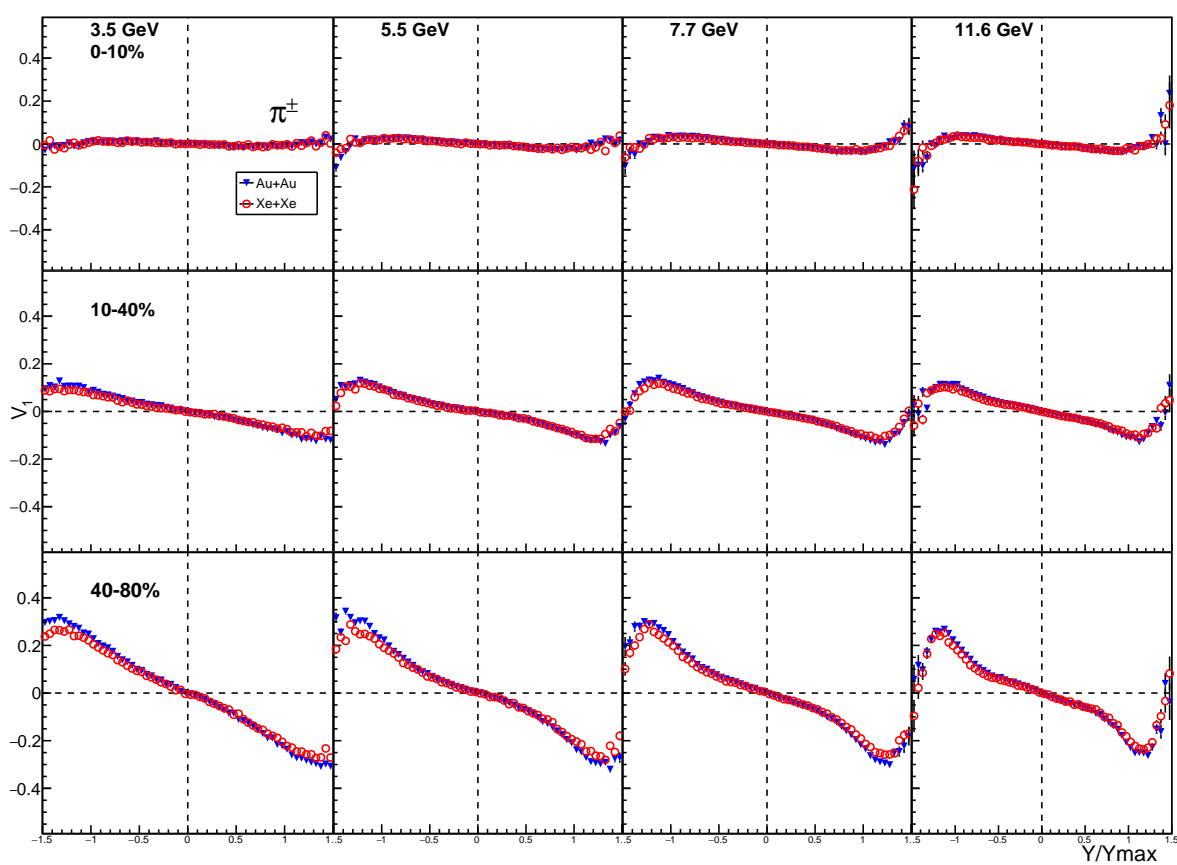

Figure 2: The same as Fig. 1 but for the directed flow of charged pions. 
[19]. We consider directed flow of protons, $v_{1}^{p}(y)$, first. These distributions calculated within the QGSM are displayed in Fig. 1.

For the peripheral collisions directed flow of protons demonstrates weak antiflow at midrapidity. In the fragmentation regions $v_{1}^{p}(y)$ is always positive. The antiflow area quickly shrinks with decreasing energy of nuclear collisions. In the reduced variable, $y^{*}=y / y_{\text {proj }}$, there is almost no difference between the distributions in $\mathrm{Au}+\mathrm{Au}$ and $\mathrm{Xe}+\mathrm{Xe}$ collisions. For the collisions with centrality $0-10 \%$ and $10-40 \%$ we sees weak antiflow at $\sqrt{s}=19.6 \mathrm{GeV}$, however, at energies below $\sqrt{s}=7.7 \mathrm{GeV}$ the directed flow of protons is already normally elongated. This is happening because the flying away baryon-rich remnants of colliding nuclei are closer to the midrapidity zone, and the directed flow of charged hadrons in the remnants is developed in normal direction. Note also that the transition from antiflow to normal flow occurs at lower energies in heavy-ion collisions compared to the light-ion ones. The explanation of this effect is as follows [11, 12, 13]. Hadrons are produced more copiously in heavy-ion collisions than in light-ion collisions at the same centrality range. Hadrons emitted in the direction of nuclear remnants will interact further thus acquiring an extra momentum. These hadrons will be pushed from the midrapidity area to higher rapidity regions. For a light-ion system with lower multiplicity of secondary hadrons the lost of few hadrons emitted in normal flow direction would be more noticeable compared to the heavy-ion system. It is worth noting that this effect is opposite to the softening of the directed flow caused by the formation of quark-gluon plasma. The QGP is expected to be produced in central and semicentral heavy-ion collisions rather than in the light-ion ones. Therefore, the softening of proton directed flow at $y=0$ should occur earlier in $\mathrm{Au}+\mathrm{Au}$ collisions compared to collisions of $\mathrm{Xe}+\mathrm{Xe}$ or lighter nuclei at the same bombarding energy.

For the directed flow of pions, shown in Fig. 2, the situation is more stable. $v_{1}^{\pi}$ at midrapidity demonstrates a distinct antiflow behavior irrespective of the colliding energy and masses of colliding nuclei. Pion antiflow becomes stronger as the reaction becomes more peripheral. However, directed flow of pions possesses another peculiarity, - it changes sign for pions with high transverse momentum. Figures 3 and 4 display the directed flow of protons and pions, respectively, in UrQMD calculated $\mathrm{Au}+\mathrm{Au}$ and $\mathrm{Xe}+\mathrm{Xe}$ collisions at $\sqrt{s}=5.5 \mathrm{GeV}$. Two distributions are shown in each plot, one for hadrons with $p_{T}>0.2 \mathrm{GeV} / c$, and another for hadrons with $p_{T}>0.9 \mathrm{GeV} / c$. For protons the difference is negligibly small, see Fig. 3. But pions from central and semicentral collisions start to develop almost zero flow (in $\mathrm{Xe}+\mathrm{Xe}$ ) and very weak antiflow (in $\mathrm{Au}+\mathrm{Au}$ ). The same tendency can be observed in QGSM calculations of the same distributions, depicted in Figs. 5 and 6 . Here the directed flow of pions seems to change its development more drastically even for very peripheral collisions, as compared to the UrQMD, however, the vertical scales of two plots are different, and the real difference between the two models is not so dramatic. Note also, that these peculiarities of the flow development arise naturally in both microscopic models without tuning of the free parameters.

Then, Fig. 7 (upper row) presents the snapshot of particle directed flows and densities for protons, antiprotons and charged pions in UrQMD calculations of $\mathrm{Au}+\mathrm{Au}$ collisions with the impact parameter $b=6 \mathrm{fm}$ at $\sqrt{s}=7.7 \mathrm{GeV}$ after $10 \mathrm{fm} / c$ of beginning of the collision. The whole space was subdivided into cells with volume $V=3 \mathrm{fm}^{3}$. The arrows indicate the collective velocities calculated for each cell. Density contours show that spatial distributions of $p, \bar{p}$ and $\pi^{ \pm}$are quite different. Protons are strongly influenced by the spectators, whereas the distributions of pions and 


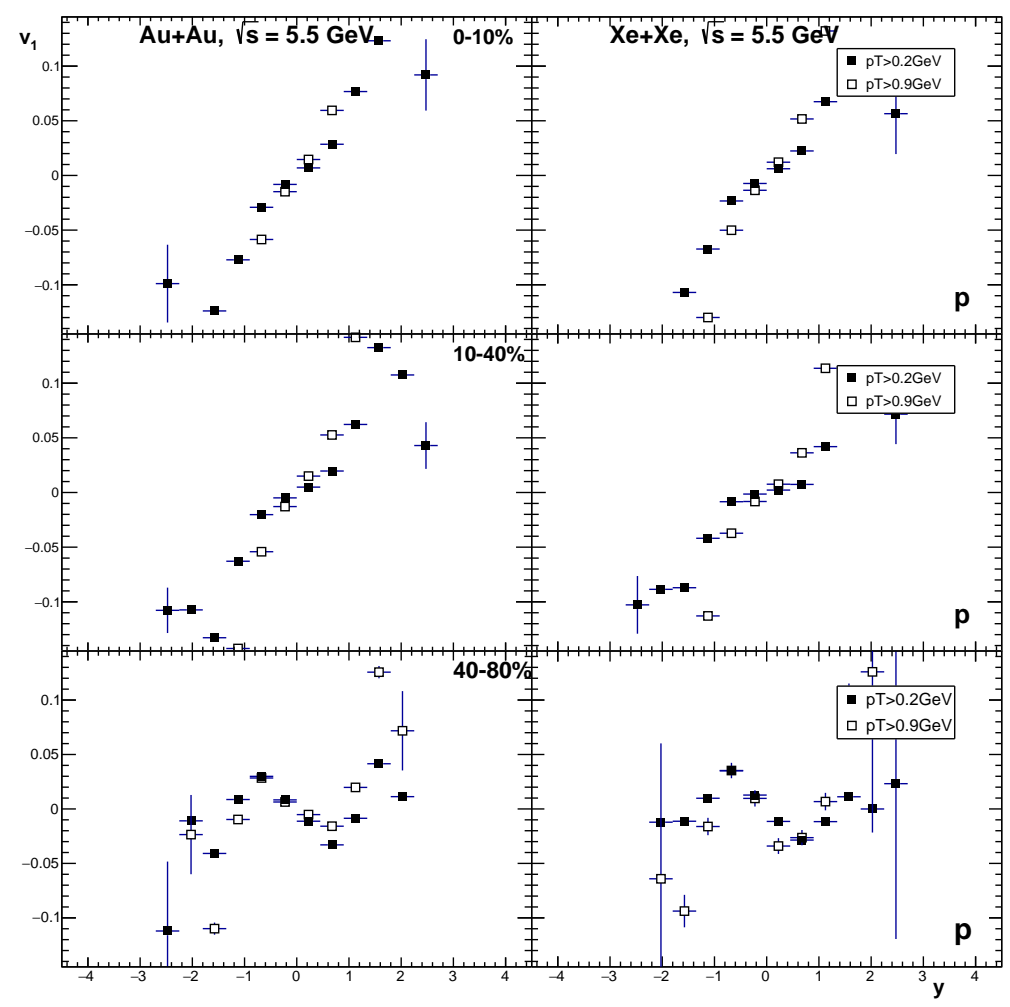

Figure 3: Directed flow of protons vs rapidity in UrQMD calculations of $\mathrm{Au}+\mathrm{Au}$ (left column) and $\mathrm{Xe}+\mathrm{Xe}$ (right column) collisions at $\sqrt{s}=5.5 \mathrm{GeV}$ with centrality $0-10 \%$ (upper row), $10 \%-40 \%$ (middle row) and $40 \%-80 \%$ (bottom row), respectively. Full symbols denote protons with $p_{T}>0.2 \mathrm{GeV} / c$, open symbols are related to protons with $p_{T}>0.9 \mathrm{GeV} / c$.

antiprotons are more symmetric w.r.t. $z=0$. The arising directed flow is a result of superposition of the partial flows in the cells, each having either positive (normal flow) or negative (antiflow) sign. Evolution of the directed flow of these three particle species at midrapidity is displayed in Fig. 5 also (bottom row). Although both normal flow and antiflow of all species are quickly developed within first $1.5 \mathrm{fm} / c$, their resulting flow is weak compared to both, flow and antiflow, components. It looks like the development of directed flow takes time longer than $t=14 \mathrm{fm} / c$ even at midrapidity.

Development of directed flow, as well as any other flow component, in the microscopic models cannot be studied alone without the analysis of the freeze-out conditions. Both UrQMD and QGSM do not support the picture of sudden freeze-out. In contrast, the models demonstrate the continuous freeze-out of hadrons, similar to that displayed in Fig. 8. Here $d N / d t$ distribution of protons, charged pions, charged kaons and Lambdas is calculated within the UrQMD for very central $\mathrm{Au}+\mathrm{Au}$ collisions at $\sqrt{s}=7.7 \mathrm{GeV}$. Arrows show the average emission times of the hadron species. According to this figure, the freeze-out of particles is continuous and not uniform. Mesons are frozen earlier than baryons, and thus the flows carried by these particles should be traced back to the flow of the cells from which the hadrons were emitted. This non-trivial task needs further investigations. 


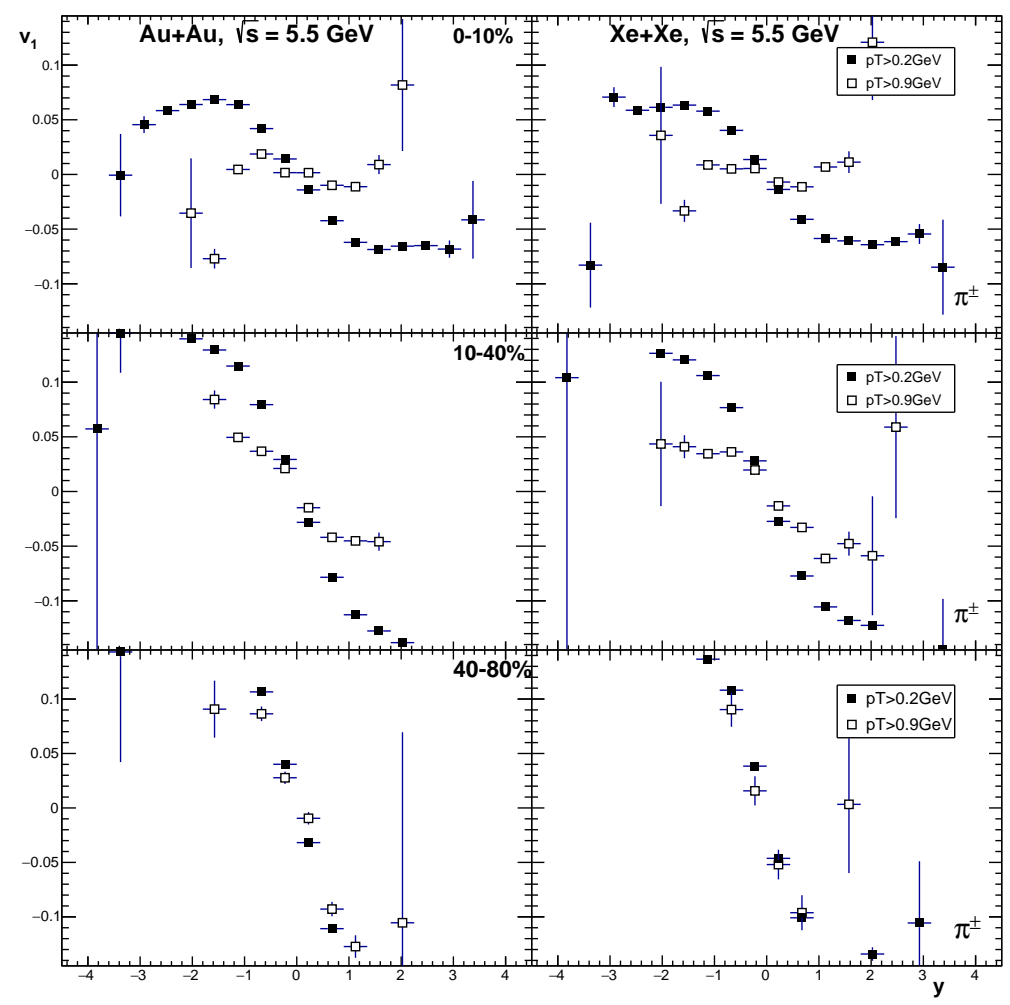

Figure 4: The same as Fig. 3 but for the directed flow of charged pions.

\section{Conclusions}

General features of directed and elliptic flows of hadrons produced in relativistic heavy-ion collisions are studied within two microscopic Monte Carlo models. The following conclusions can be drawn. Both models favor the reduction of proton directed flow at midrapidity in heavy-ion collisions with (i) increasing energy of collisions (from $\sqrt{s}=3.5 \mathrm{GeV}$ ) and (ii) increasing impact parameter of collisions. This effect, which is caused by hadronic rescattering in remnants of the nuclei, can mimic creation of the quark-gluon plasma. However, the so-called softening of the directed flow due to rescattering is stronger in light- and intermediate-ion collisions compared to the heavy-ones. In case of the QGP formation the effect should be opposite. Directed flows of mesons and antibaryons are negative (or close to zero) for light- and heavy-ion collisions in the considered energy range. Our study suggests also that the flow development should be investigated together with the hadronic freeze-out.

\section{Acknowledgments}

This work was supported in parts by the Russian Foundation for Basic Research under grant No. 18-02-40084 and grant No. 18-02-40085, and by the Norwegian Centre for International Cooperation in Education (DIKU) under grants "CPEA-LT-2016/10094 - From Strong Interacting Matter to Dark Matter" and "UTF-2016-long-term/10076 - Training of Bachelor, Master and PhD Students specialized in high energy physics". 


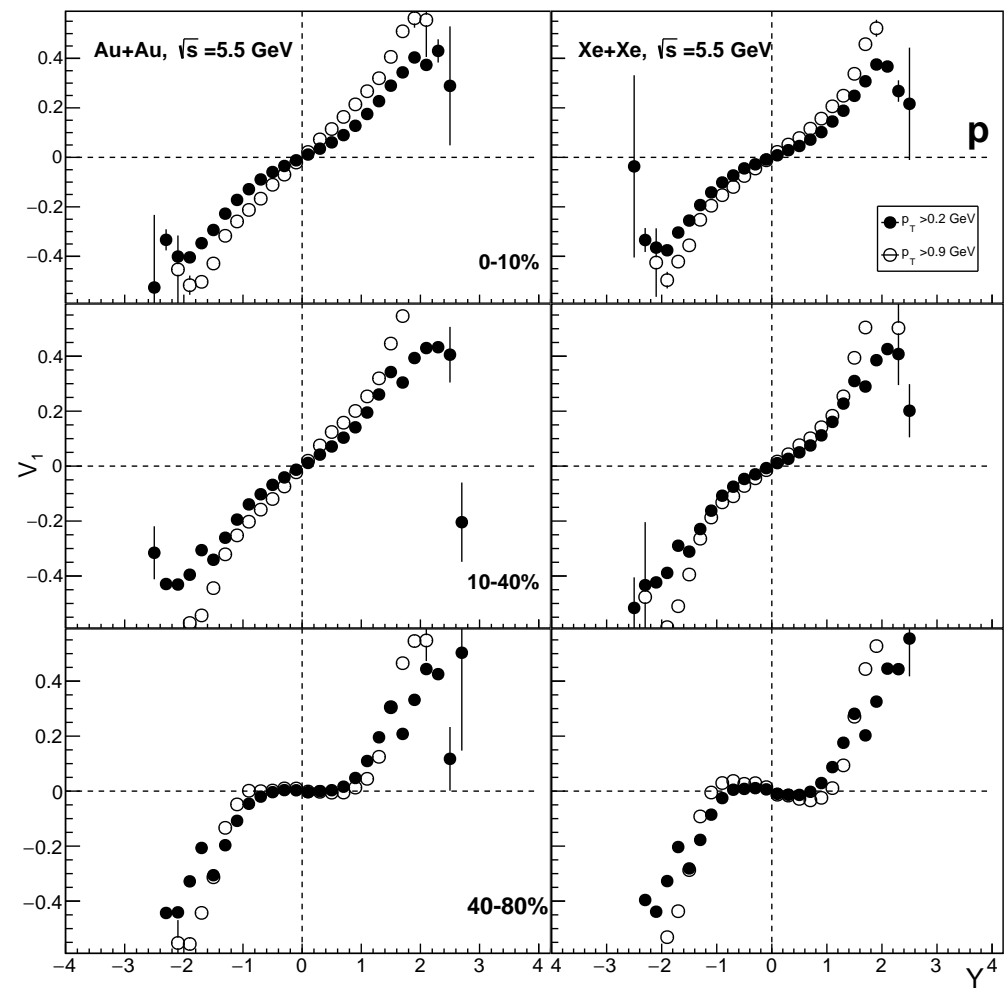

Figure 5: The same as Fig. 3 but for the directed flow of protons in QGSM calculations.

\section{References}

[1] S. Voloshin and Y. Zhang, Flow study in relativistic nuclear collisions by Fourier expansion of azimuthal particle distributions, Z. Phys. C 70, 665 (1996).

[2] A.M. Poskanzer and S.A. Voloshin, Methods for analyzing anisotropic flow in relativistic nuclear collisions, Phys. Rev. C 58, 1671 (1998).

[3] W. Reisdorf and H.G. Ritter, Collective flow in heavy-ion collisions, Ann. Rev. Nucl. Part. Sci. 47, 663 (1997).

[4] C.M. Hung and E.V. Shuryak, Hydrodynamics near the QCD phase transition: Looking for the longest lived fireball, Phys. Rev. Lett. 75, 4004 (1995)

[5] L.P. Csernai and D. Rohrich, Third flow component as QGP signal, Phys. Lett. B 458, 454 (1999)

[6] J. Brachmann et al., Antiflow of nucleons at the softest point of the EoS, Phys. Rev. C 61, 024909 (2001)

[7] Yu.B. Ivanov and A.A. Soldatov, Directed flow indicates a cross-over deconfinement transition in relativistic nuclear collisions, Phys. Rev. C 91, 024915 (2015)

[8] L.V. Bravina, Scaling violation of transverse flow in heavy ion collisions at AGS energies, Phys. Lett. B 344, 49 (1995)

[9] L. Adamczyk et al. (STAR Collaboration), Beam-Energy Dependence of the Directed Flow of Protons, Antiprotons, and Pions in Au+Au Collisions, Phys. Rev. Lett. 112, 162301 (2014) 


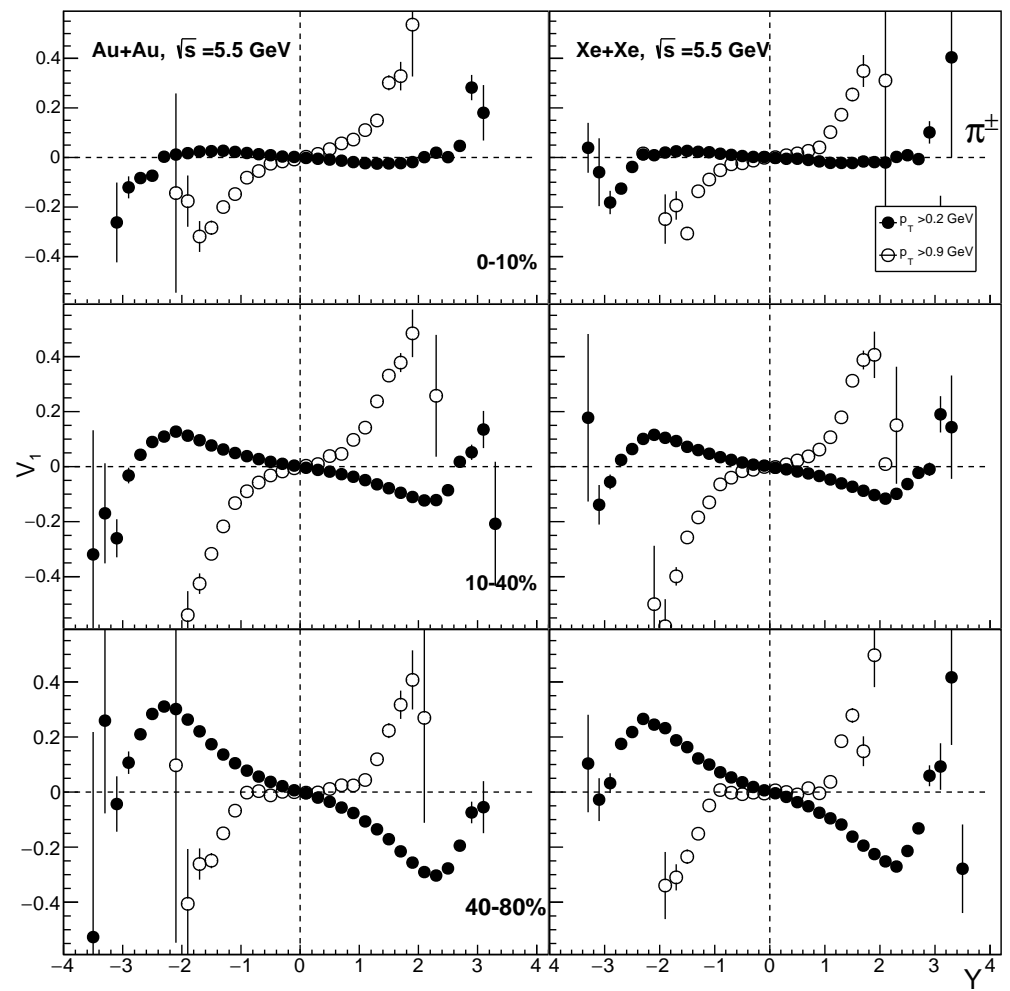

Figure 6: The same as Fig. 5 but for the directed flow of charged pions.
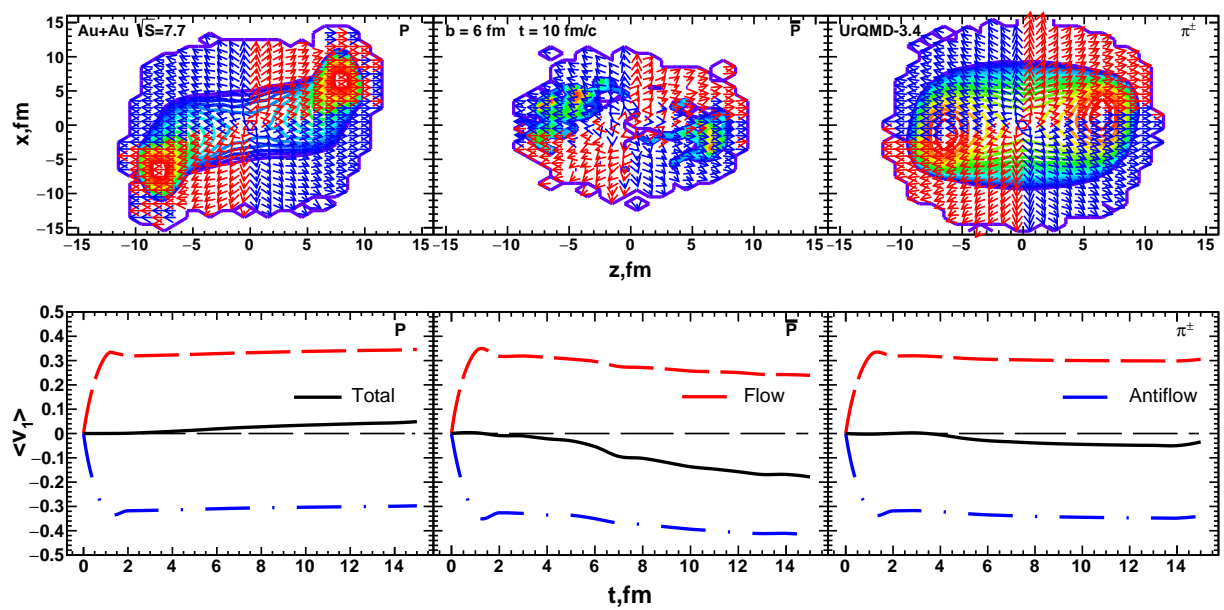

Figure 7: Upper row: The snapshot at time $t=10 \mathrm{fm} / c$ of hadron densities (contour plots) and collective velocities (arrows) of the cells, each with volume $V=3 \mathrm{fm}^{3}$, for protons (left), antiprotons (middle), and charged pions (right) in UrQMD calculations of Au+Au collisions at $\sqrt{s}=7.7 \mathrm{GeV}$ with $b=6$ fm. Bottom row: The time development of total directed flow (solid line) and partial flows in normal flow (red dashed line) and antiflow (blue dash-dotted line) directions of protons (left), antiprotons (middle), and charged pions (right) in these reactions. 


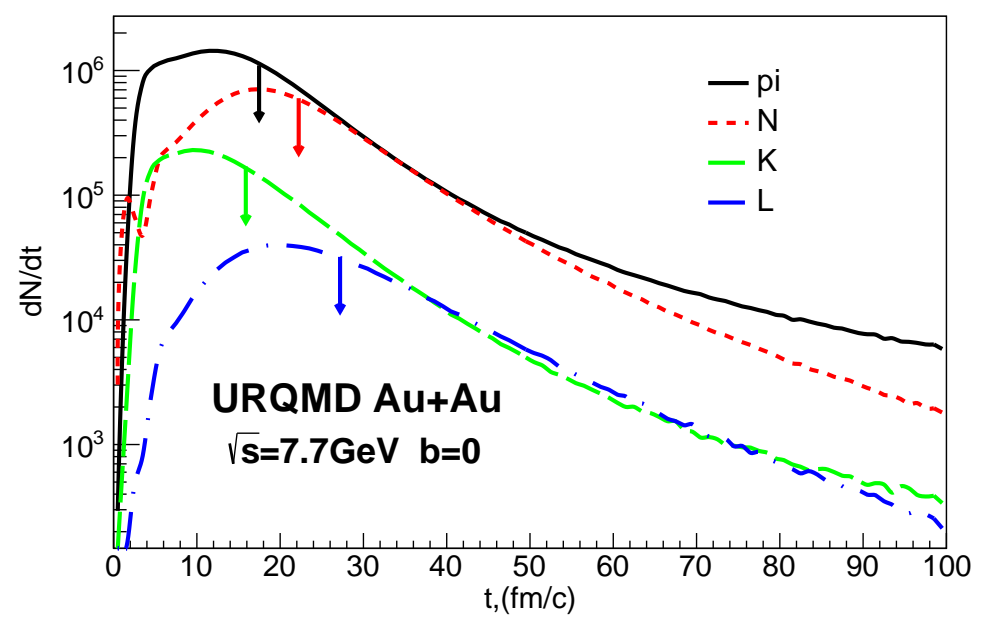

Figure 8: $d N / d t$ distribution of the particles over their last collision time $t$ for kaons (dashed), pions (solid), nucleons (dotted) and lambdas (dash-dotted histograms) for Au+Au collisions at $\sqrt{s}=7.7 \mathrm{GeV}$ at $b=0$. The vertical arrows correspond to the average emission times of the species.

[10] L. Adamczyk et al. (STAR Collaboration), Beam-Energy Dependence of Directed Flow of $\Lambda, \bar{\Lambda}, K^{ \pm}, K_{s}^{0}$ and $\phi$ in Au+Au Collisions, Phys. Rev. Lett. 120, 062301 (2018)

[11] L.V. Bravina, Amand Faessler, C. Fuchs, E.E. Zabrodin, Microscopic study of energy and centrality dependence of transverse collective flow in heavy ion collisions, Phys. Rev. C 61, 064902 (2000)

[12] E.E. Zabrodin, C. Fuchs, L.V. Bravina, Amand Faessler, Transverse momentum dependence of directed particle flow at 160A-GeV, Phys. Rev. C 63, 034902 (2001)

[13] L.V. Bravina and E.E. Zabrodin, Directed flow in heavy-ion collisions at NICA: What is interesting to measure?, Eur. Phys. J. A 52, 245 (2016)

[14] S.A. Bass et al., Microscopic models for ultrarelativistic heavy ion collisions, Prog. Part. Nucl. Phys. 41, 255 (1998)

[15] M. Bleicher et al., Relativistic hadron hadron collisions in the ultrarelativistic quantum molecular dynamics model, J. Phys. G 25, 1859 (1999)

[16] N.S. Amelin and L.V. Bravina, The Monte Carlo Realization of Quark-Gluon String Model for Description of High-energy Hadron Hadron Interactions, Sov. J. Nucl. Phys. 61, 133 (1990)

[17] J. Bleibel, L.V. Bravina, E.E. Zabrodin, How many of the scaling trends in pp collisions will be violated at $\sqrt{s_{N N}}=14 \mathrm{TeV}$ ? - Predictions from Monte Carlo quark-gluon string model, Phys. Rev. D 93, 114012 (2016)

[18] L.V. Bravina, Yu. Kvasiuk, S.Yu. Sivoklokov, O. Vitiuk, E.E. Zabrodin, Anisotropic (v1 and v2) flow in relativistic heavy-ion collisions at energies between $4 \mathrm{GeV}$ and $200 \mathrm{GeV}$, EPJ Web Conf. 191, 05004 (2018)

[19] L.V. Bravina, Yu. Kvasiuk, S.Yu. Sivoklokov, O. Vitiuk, E.E. Zabrodin, Directed flow in microscopic models in relativistic A+A collisions, Universe 5, no.3, 69 (2019) 